\title{
Editorial
}

\section{BARRERAS PARA EL ACCESO A SERVICIOS DE SALUD BUCAL.}

Los países de Latinoamérica han dado un gran paso en el acceso universal a la salud y la cobertura universal de la misma, en la XXX reunión de RESSCAD en El Salvador en el 2014; se presentó la estrategia para este fortalecimiento en los países y sus sistemas de salud en la región. En el marco de esa misma reunión se presentó el documento aprobado de la Organización Panamericana de la Salud, donde se define este concepto de la siguiente manera: "El acceso universal a la salud implica la eliminación de las barreras de acceso geográfico, cultural y financieras. La cobertura universal de salud se construye sobre el acceso universal a la salud permitiendo la utilización de los servicios de salud de calidad cuando se necesitan" ${ }^{1}$.

Respecto a estas tres dimensiones, la carrera de odontología de la Universidad Católica de Cuenca, en el año 2017 ha iniciado una macro investigación poblacional, en cuanto a las barreras culturales, en la sub dimensión barreras psico-socio-culturales ${ }^{2}$,con la finalidad de estudiar la alfabetización en salud bucal, la indiferencia al cuidado odontológico, la negligencia al cuidado dental, la automedicación en odontología y la ansiedad al tratamiento dental, como barreras escalonadas y progresivas respecto al acceso a servicios de salud bucal.

La alfabetización en salud bucal, es el grado en que los individuos tienen la capacidad de obtener, procesar y comprender información y servicios de salud bucal necesarios para tomar decisiones de salud apropiadas ${ }^{3}$, es el primer nivel de barrera.

Mientras que la indiferencia al cuidado odontológico, es un constructo que se refiere a una actitud que consiste en una infravaloración de la importancia de los dientes y la falta de interés en la salud bucal que se manifiesta tanto en la negligencia al cuidado bucal, como en el cumplimiento deficiente de las recomendaciones de cuidado bucal y una actitud de solución rápida a los problemas de salud buco dental como también una actitud negativa para asistir al consultorio odontológico, que no se deba a ansiedad al tratamiento ${ }^{4}$, esta corresponde a un segundo nivel de barrera para el acceso a servicios de salud bucal.

El tercer nivel de barrera lo representa la negligencia al cuidado dental, que se entiende como

\footnotetext{
${ }^{1}$ Organización Panamericana de la Salud. El acceso universal a la salud y la cobertura universal de salud es el compromiso de toda la sociedad para promover la salud y el bienestar. [Online].; 2014. Disponible en http://www.paho.org/

${ }^{2}$ Landini E, González-Cowes V, Damore E. Hacia un marco conceptual para repensar la accesibilidad cultural. Cad. Saúde Pública, Rio de Janeiro. 2014;: p. 231-244.

${ }^{3}$ Lee J, Divaris K, Baker A, Rozier R, Vann W. The relationship of oral health literacy and self-efficacy with oral health status and dental neglect. Am J Public Health. 2012 Mayo; 102(5)

${ }^{4}$ Nuttall N. Initial development of a scale to measure dental indifference. Community Dent Oral Epidemiol. 1996 Abril; 24(2).
} 
la falta de tomar precauciones para mantener la buena salud bucodental, la falta de buscar atención dental necesaria y el descuido físico de la cavidad bucal ${ }^{5}$, a pesar de que se sabe que se necesita. Cuando la persona percibe la necesidad de atención a un problema de su salud bucal y recurre al "consumo de medicamentos, hierbas y remedios caseros por propia iniciativa o por consejo de otra persona, sin consultar al médico" ${ }^{6}$ se denomina auto medicación, se consideró como la cuarta barrera de acceso a servicios de salud bucal.

Finalmente, la ansiedad al tratamiento dental, que se define como la respuesta del paciente al estrés específico de la situación de tener que recibir atención odontológica ${ }^{7}$, fue considerada como la última barrera para el acceso a servicios de salud bucal.

Dr. Ebingen Villavicencio Caparó Director de la Revista

\footnotetext{
${ }^{5}$ Jamieson L, Thomson W. The Dental Neglect and Dental Indifference scales. Community Dent Oral Epidemiol. 2002 Enero; 30(3).

${ }^{6}$ Conhi A, Castillo-Andamayo DE, Del-Castillo-López C. Automedicación odontológica de pacientes que acuden a una institución pública y privada, Lima-Perú. Rev Estomatol Herediana. 2015 Jul-Set; 25(3).

${ }^{7}$ Corah N, Gale E, Illig S. Assessment of a dental anxiety scale. JADA. 1978 Noviembre; 97.
} 